\title{
Light and Electron Microscopic Study of Soft Bone Osteocytes From a Triceratops horridus Supraorbital Horn
}

\author{
Mark H. Armitage ${ }^{1}$, Kevin L. Anderson ${ }^{2}$. \\ ${ }^{1}$ CRS Microscopy Laboratory, Thousand Oaks, CA, USA. \\ 2. Department of Biology, Arkansas State University Beebe, Beebe, AR, USA.
}

Soft blood vessels, cell-like microstructures, intracellular nucleus-like spheres, primary and secondary filipodia and cell to cell junctions, as well as other cellular details, have been observed in dinosaur bones representing many different species including Tarbosaurus bataar, Tyrannosaurus rex, Brachylophosaurus canadensis, and Triceratops horridus. [1, 2, 3, 4, 5].

We reported soft sheets of fibrillar bone tissues from an intact Triceratops horn from the Hell Creek Formation in MT [6]. We illustrated red blood cell (RBC) like microstructures, oblate and stellate osteocytes, and organelle-like components within osteocytes. Soft vessels were found protruding from fractured Triceratops ribs, but no soft vessels were found in the horn. Our findings are significant and unique due to the decomposed nature of the horn and the presence of wet matrix, fungal hyphae (Figure 1), plant roots (Figure 2) and the ubiquitous presence of microorganisms.

The remarkable preservation of delicate ultrastructures such as filipodia (Figure 3), has defied explanation despite hypothesized temporal limits on molecular preservation in the hundreds of thousands of years [7]. In the case of soft vessels recovered from dinosaur femur specimens, it seems reasonable that tissues were sequestered from the elements and from biological activity because of encapsulation within compact bone. Within the highly vascular Triceratops horn, however, no sequestration was likely, yet a high degree of preservation persists.

Recent work suggests that the iron rich nanoparticles synthesized by ferritin in living systems produces insoluble, mineralized rust/iron after death and that (by a rudimentary cross-linking), the insoluble iron "fixes" tissues like formaldehyde fixatives [7]. However only one study has investigated this, and it examined tissue preservation for a just few months. It was performed on ostrich vessels bathed in concentrated, purified hemoglobin (all cells and plasma removed) extracted from red blood cells and left at room temperature on a bench - not very representative of conditions that dinosaur remains must have been subjected to at Hell Creek [7]. Moreover, the mineralized iron is shown to be encapsulated in a layer embedded within vessel tissues. It is unclear how a layer of iron will fix tissues not in intimate proximity to the layer.

In this report, we show high magnification evidence of ultrastructures preserved within osteocytes from Triceratops horn material, even in the absence of iron nanoparticles (Figure 4). Osteocyte filipodia are smooth and elongated structures and show no ruffled border that would be associated with iron nanoparticles adsorbed to the surface. Similarly, osteocyte internal, spherical, nucleus-like microstructures show no rough border that might indicate the presence of iron nanoparticles (Figure 5). In contrast, round, RBC-like microstructures filling the lumen of a Triceratops horn vessel exhibit a rough border and are likely covered in iron nanoparticles (Figure 6). 

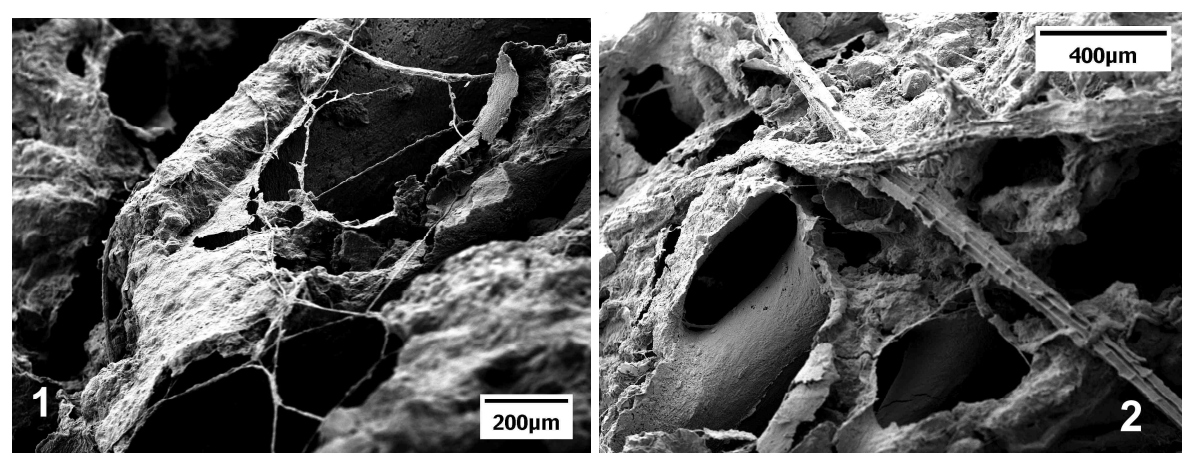

Figure 1. Fungal hyphae in horn. Figure 2. Roots in horn.

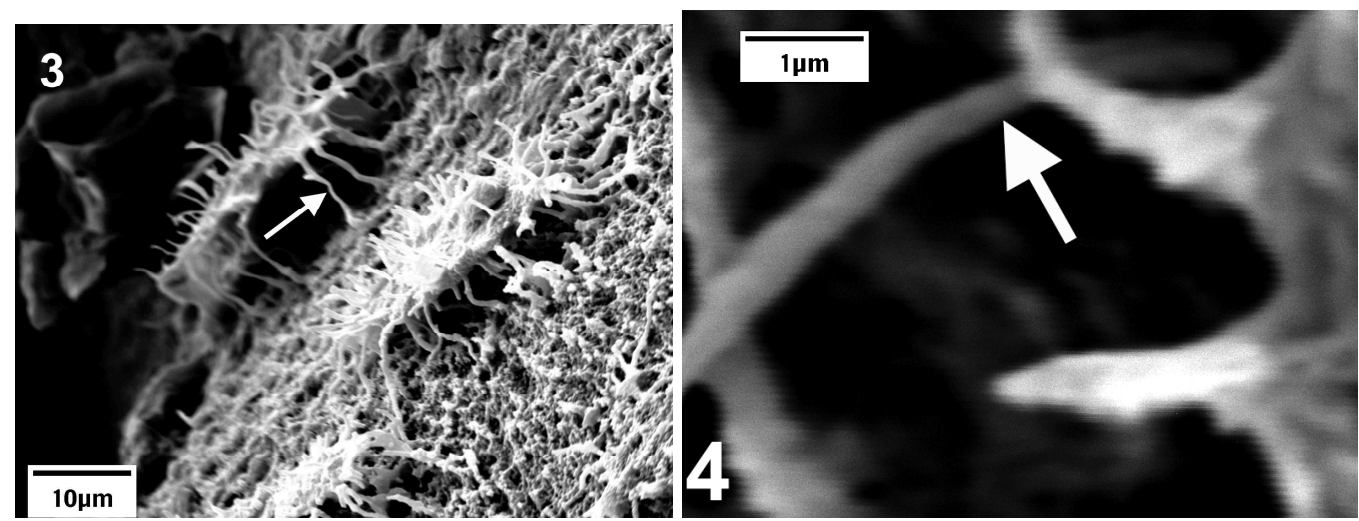

Figure 3. Elongated, smooth filipodia (arrow). Figure 4. Elongated, smooth filipodia (arrow).

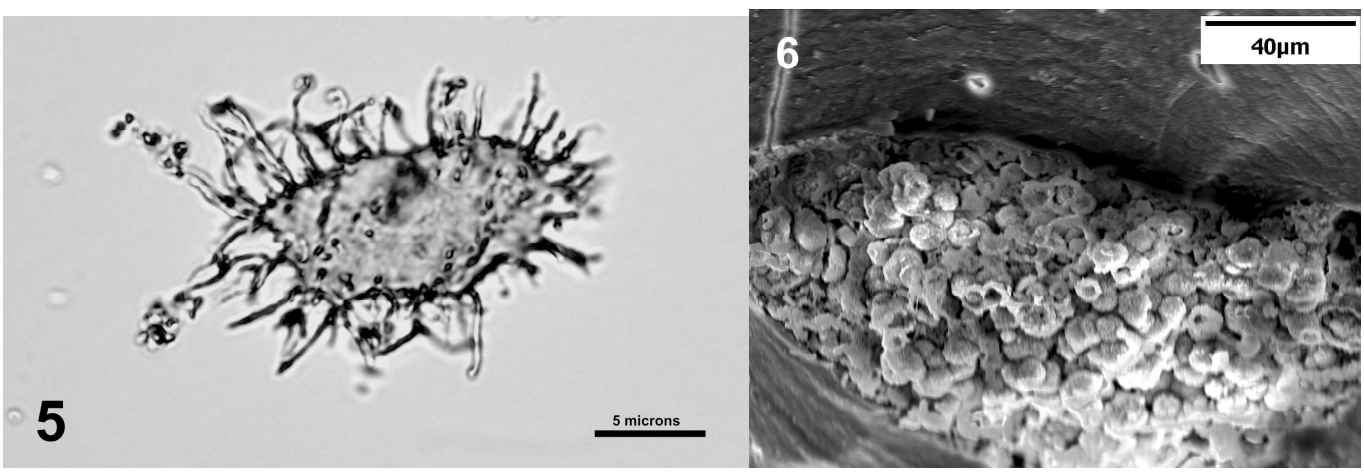

Figure 5. Osteocyte with round nucleus-like structure. Figure 6. RBC-like structures in vessel.

[1] R Pawlicki, Acta Anat. 100 (1978), p. 411-418.

[2] R Pawlicki and M Nowogrodzka-Zagorska, Ann Anat 180 (1998), p. 73-77.

[3] MH Schweitzer and J Horner, Ann Paleon 85 (1999), p. 179-192.

[4] MH Armitage, Crea Res Soc Quart 38 (2001), p. 61-66.

[5] L Zylberberg and M Laurin, C R Paleovol 10 (2011), p. 357-366.

[6] MH Armitage and KL Anderson, Acta Histochemica 115 (2013), p. 603-608.

[7] MH Schweitzer et al. Proc Royal Sci B 281 (2014), p. 3-10. 